\title{
Effects of an oral synbiotic on the gastrointestinal immune system and microbiota in patients with diarrhea-predominant irritable bowel syndrome
}

\author{
Adrian Mathias Moser ${ }^{1,2} \cdot$ Walter Spindelboeck ${ }^{1,2} \cdot$ Bettina Halwachs $^{2,3} \cdot$ Heimo Strohmaier ${ }^{4}$ Patrizia Kump ${ }^{1,2}$. \\ Gregor Gorkiewicz $^{2,3}$. Christoph Högenauer ${ }^{1,2}$
}

Received: 21 February 2018 / Accepted: 18 September 2018 / Published online: 24 September 2018

(c) The Author(s) 2018

\begin{abstract}
Purpose Diarrhea-predominant irritable bowel syndrome (IBS-D) is a common functional gastrointestinal disorder. Probiotics and synbiotics have been shown to improve symptoms of IBS, although mechanisms of action are currently not understood.

Methods We investigated the effects of a 4-week oral synbiotic treatment (OMNi-BiOTiC ${ }^{\circledR}$ Stress Repair) in ten IBS-D patients on gastrointestinal mucosal and fecal microbiota, mucosa-associated immune cells, and fecal short-chain fatty acids. The upper and lower gastrointestinal tracts were compared before and after a 4-week synbiotic treatment using endoscopic evaluation to collect mucosal specimens for FACS analysis and mucosal 16S rRNA gene analysis. In stool samples, analysis for fecal SCFAs using GC-MS, fecal zonulin using ELISA, and fecal 16S rRNA gene analysis was performed.

Results Synbiotics led to an increased microbial diversity in gastric $(p=0.008)$ and duodenal $(p=0.025)$ mucosal specimens. FACS analysis of mucosal immune cells showed a treatment-induced reduction of CD4 ${ }^{+} \mathrm{T}$ cells $(60 \mathrm{vs} .55 \%, p=0.042)$ in the ascending colon. Short-chain fatty acids (acetate $101 \mathrm{vs.} 202 \mu \mathrm{mol} / \mathrm{g} ; p=0.007$ ) and butyrate ( $27 \mathrm{vs.} 40 \mu \mathrm{mol} / \mathrm{g} ; p=0.037$ ) were elevated in fecal samples after treatment. Furthermore, treatment was accompanied by a reduction of fecal zonulin concentration ( 67 vs. $36 \mathrm{ng} / \mathrm{ml} ; p=0.035$ ) and disease severity measured by IBS-SSS ( 237 vs. $54 ; p=0.002$ ).

Conclusions Our findings indicate that a short-course oral synbiotic trial may influence the human gastrointestinal tract in IBS-D patients on different levels which are region specific.
\end{abstract}

Keywords Irritable bowel syndrome $\cdot$ Microbiota $\cdot$ Short-chain fatty acids $\cdot$ Mucosal immune cells

Adrian Mathias Moser and Walter Spindelboeck contributed equally.

Electronic supplementary material The online version of this article (https://doi.org/10.1007/s00394-018-1826-7) contains supplementary material, which is available to authorized users.

Christoph Högenauer

christoph.hoegenauer@medunigraz.at

Adrian Mathias Moser

adrianm.moser@hotmail.com

Walter Spindelboeck

walter.spindelboeck@medunigraz.at

Bettina Halwachs

bettina.halwachs@medunigraz.at

Heimo Strohmaier

heimo.strohmaier@medunigraz.at

Patrizia Kump

patrizia.kump@medunigraz.at
Gregor Gorkiewicz

gregor.gorkiewicz@medunigraz.at

1 Division of Gastroenterology and Hepatology, Department of Internal Medicine, Medical University of Graz, Auenbruggerplatz 15, 8036 Graz, Austria

2 Theodor Escherich Laboratory for Microbiome Research, Medical University of Graz, Auenbruggerplatz 15, 8036 Graz, Austria

3 Institute of Pathology, Medical University of Graz, Auenbruggerplatz 25, 8036 Graz, Austria

4 Center for Medical Research, Medical University of Graz, Stiftingtalstraße 24, 8010 Graz, Austria 


\section{Introduction}

Irritable bowel syndrome (IBS) is a functional disorder of the gastrointestinal (GI) tract. Since the global prevalence of IBS is estimated to be between 10 and 20\% depending on diagnostic criteria and geographic region, it constitutes a major medical burden and is thereby considered a public health issue [1]. The subtype diarrhea-predominant IBS (IBS-D) reflects diarrhea as the predominant symptom. Diagnosis is made by the symptom-based ROME criteria after the exclusion of organic disease [2]. To date, the treatment of IBS-D is focused on relief of symptoms rather than cure and consists of nutritional interventions, psychological or medical therapies [3].

Intestinal mucosal inflammation is a hallmark of disease in IBS-D [4, 5]. Specific compositional changes in the microbiota are thought to trigger these mucosal inflammatory responses in IBS-D which in turn are able to stimulate visceral hypersensitivity and pain $[3,4,6,7]$. Thereby, the possible dysbiosis-triggered mucosal inflammation involves elevated pro-inflammatory cytokines that can interact with colonic nociceptive and non-nociceptive afferent nerves. If activated, these afferent nerves can sensitize mechanosensory colonic c-fibers to mediate pain symptoms in IBS-D patients [4]. Furthermore, activated mast, B and plasma cells accompanied with an impaired barrier function in jejunal specimens are present in IBS-D patients and the grade of mucosal inflammation is thereby associated to clinical disease activity. These microbiotaneuro-immunological interactions might, therefore, be involved in triggering intestinal hypersensitivity and pain, suggesting that IBS-D has a microbiota-dependent immune-mediated pathogenesis [8-10].

Therapies for IBS-D influencing the composition and function of the intestinal ecosystem are, therefore, of interest although mechanisms of action of probiotic (preparations with microorganisms only), prebiotic (food ingredients that enhance the growth of specific microorganism, e.g., inulin, starch), and synbiotic (combination of pre-and probiotics) therapies are not fully understood. However, extensive research is ongoing to clarify the role of specific probiotic strains and formulations on the host [11-13]. A recent meta-analysis including several oral probiotics in IBS suggests a clinical efficacy with significant decrease in various IBS-related symptoms after treatment [14]. The proposed mechanisms of action of different orally given probiotic strains are influences on mucosal immune cells, e.g., the IL-10 inducing effect of Faecalibacterium prausnitzii in dendritic cells (DCs) and subsequent modulation of regulatory $\mathrm{T}$ cells (Tregs). Furthermore, a mix of Clostridia strains stimulated mucosal Tregs, Lactobacillus rhamnosus had direct impacts on the mucosal barrier and Bifidobacterium bifidum changed the gut microbiota composition [15-18]. In IBS, different types of probiotics showed influences on fecal and mucosal microbiota composition as well as intestinal barrier function, but systematic investigation of the effects on different regions of the GI tract and the mucosal immune system is lacking [19-21]. Furthermore, Bifidobacterium bifidum has been shown to influence the level of fecal short-chain fatty acids (SCFAs) in humans, which are known to play an important role in mediating gastrointestinal homeostasis [18, 22]. However, data on clinical efficacy and mechanisms of action of synbiotics in IBS, especially regarding possible region-specific differences in the upper and lower GI tracts, remain to be scarce [23]. To elucidate which regions and levels of the intestinal ecosystem could be involved in mechanisms of action of synbiotics in IBS-D, we investigated the GI tract in ten patients with IBS-D before and after a 4-week treatment with an open-label oral synbiotic mixture (containing multiple species of probiotic strains and prebiotics including inulin, starch, and fructooligosaccharides). The effects of synbiotic treatment were determined by exploring mucosal immune cells (sampled in duodenum and ascending colon), SCFAs, and zonulin (a surrogate for the intestinal barrier function [24]), from stool and the gastrointestinal microbiota (from stool as well as from mucosal samples originating from stomach, duodenum, and colon).

\section{Methods}

We investigated the effects of a 4-week oral synbiotic on ten IBS-D patients by comparing the following parameters preand post-treatment. Patients were examined by endoscopic evaluation of the upper and lower gastrointestinal tracts to obtain mucosal samples for FACS analysis and mucosal $16 \mathrm{~S}$ rDNA analysis. Thereby, biopsies for FACS analysis were separately taken from the duodenum and ascending colon during the retraction of the endoscope and immediately processed. The colonic biopsies were obtained between the right-colonic flexure and the caecum. In addition, biopsies from the gastric corpus, duodenum, and ascending colon were obtained for mucosal microbiota analysis. Furthermore, analysis of fecal SCFAs, zonulin, and fecal 16S rDNA was performed.

\section{Patients and controls}

IBS-D patients (according to S3 guidelines) for this study were recruited consecutively from the outpatient clinics of the Department of Internal Medicine, Division of Gastroenterology and Hepatology, Medical University of Graz [25]. The following inclusion criteria were applied: (1) 
symptomatic IBS patients according to current S3 guideline [25]; (2) age between 18 and 65 years; and (3) informed consent. Exclusion criteria were: (1) chronic inflammatory (IBD, celiac disease, and microscopic colitis were ruled out, and patients had the previous endoscopic evaluation and diagnostics), immune-, or neoplastic diseases; (2) recent application of immune-modifying medication; (3) pregnancy; and (4) alcohol or drug abuse. No patients received any new medications during the study period and no changes in medication dose were made during the study. No antibiotics were taken 4 weeks prior to study inclusion. All medications used by patients are systemically compiled in Table 1 . All individuals included signed an informed consent prior to study inclusion. All protocols and informed consents were a priori waived by the local institutional review board (IRB number IRB00002556), vote 25-594 ex 12/13.

\section{Study protocol and schedule}

Patients were recruited from the outpatient department as mentioned. During a screening visit, inclusion and exclusion criteria were applied and physical status investigated. Patients fulfilling criteria signed an informed consent and were scheduled for the baseline study visit 1. At study visit 1, upper and lower GI tract endoscopy was performed and biopsies were taken, fecal samples and IBS-SSS were obtained, and synbiotic formulation was handed out. Patients recorded the oral administration of synbiotic mixture twice a day for 4 weeks. At study visit 2 (4 weeks later), all examinations were re-performed including endoscopy and obtaining of fecal samples and IBS-SSS.

\section{IBS-SSS}

The German version of the validated IBS-SSS questionnaire was obtained from the Zentrum für klinische Ernährung (ZKES, Wollgrasweg 49b, 70599 Stuttgart, Germany) and used as described [26]. Symptoms were quantified prior and after 4 weeks of synbiotic therapy.
Table 1 Clinical and demographic data of ten patients with IBS-D included in the study

\begin{tabular}{|c|c|}
\hline Number of patients ( $\%$ female) & $10(50)$ \\
\hline Age at endoscopy, years (median [Q1-Q3]) & $46[37-53]$ \\
\hline BMI (median [Q1-Q3]) & $23[22-25]$ \\
\hline IBS-SSS baseline (median [Q1-Q3]) & 236 [129-256]; IBS diagnosis $>75$ \\
\hline \multirow[t]{8}{*}{ Relevant comorbidities (number of patients) } & Hypothyroidism (4) \\
\hline & Iron deficiency (2) \\
\hline & Depression (2) \\
\hline & Bronchial asthma (1) \\
\hline & Gastroesophageal reflux disease (1) \\
\hline & Arterial hypertension (1) \\
\hline & Osteopenia (1) \\
\hline & Osteoporosis (1) \\
\hline \multirow[t]{16}{*}{ Co-medications (number of patients) } & Thyroid hormones (4) \\
\hline & Vitamin D3 (3) \\
\hline & Proton pump inhibitor (2) \\
\hline & Calcium (2) \\
\hline & Antidepressant (2) \\
\hline & H2-blocker (1) \\
\hline & Selective estrogen receptor modulator (1) \\
\hline & Beta blocker (1) \\
\hline & Benzodiazepine (1) \\
\hline & Atypical antipsychotic (1) \\
\hline & Antacid (1) \\
\hline & Antihypertensive (1) \\
\hline & Spasmolytic (1) \\
\hline & Prokinetic (1) \\
\hline & Folate (1) \\
\hline & Vitamin B complex (1) \\
\hline
\end{tabular}

IBS-D diarrhea-predominant irritable bowel syndrome, BMI body mass index, IBS SSS irritable bowel syndrome severity scoring system 


\section{Synbiotic formulation}

Patients were given a 4-week course (twice a day) commercially available synbiotic mixture $\left(\mathrm{OMNi}-\mathrm{BiOTiC}^{\circledR}\right.$ Stress Repair, Institut Allergosan, Graz) consisting of the following prebiotics corn starch, maltodextrin, inulin, fructooligosaccharides, potassium chloride, magnesium sulfate, mangan sulfate and enzymes as well as $7.5 \times 10^{9}$ of each of the following probiotic bacterial strains: Lactobacillus casei W56, Lactobacillus acidophilus W22, Lactobacillus paracasei W20, Lactobacillus salivarius W24, Lactobacillus plantarum W62, Lactococcus lactis W19, Bifidobacterium lactis W51 and W52, and Bifidobacterium bifidum W23.

\section{Mucosal specimens}

Gastroduodeno- and ileocolonoscopy was performed with standard equipment (Olympus, Hamburg, Germany) in sedated subjects. Samples were obtained by forceps biopsy. Biopsies for FACS analysis were separately taken from the duodenum and ascending colon during the retraction of the endoscope and immediately processed. The colonic biopsies were obtained between the right-colonic flexure and the caecum. In addition, biopsies from the gastric corpus, duodenum, and ascending colon were obtained for mucosal microbiota analysis.

\section{Isolation of lamina propria mononuclear cells}

Mucosal biopsy specimens were obtained separately from the duodenum and ascending colon and immediately preserved in chilled RPMI medium (Sigma; supplemented with penicillin, streptomycin, and amphotericin). Biopsies were washed once with calcium- and magnesium-free HBSS (Life Technologies, Vienna, Austria) and then incubated in calcium- and magnesium-free HBSS containing $1 \mathrm{mM}$ DTT and $5 \mathrm{mM}$ EDTA at $37^{\circ} \mathrm{C}$ for 20 min with gentle agitation to remove mucus and epithelial cells. Following a brief wash with calcium- and magnesium-free HBSS, tissue was digested with $1 \mathrm{mg} / \mathrm{ml}$ Collagenase A (Roche, Basel, Switzerland) and 5 units/ml DNase I (Roche, Basel, Switzerland) in HBSS at $37{ }^{\circ} \mathrm{C}$ for $60 \mathrm{~min}$ on a shaker and mechanically disrupted by gentle pipetting. Complete dissociation was verified by visual inspection. After passing through a $70 \mu \mathrm{m}$ cell strainer, the released cells were washed twice with RPMI complete medium (containing $10 \%$ FCS and $1 \%$ penicillin/streptomycin) and finally resuspended in RPMI complete medium. The cell suspension was kept on ice until further analysis.

\section{Flow cytometry}

FACS analysis was performed as previously described [27-29]. Briefly, the cell suspension was washed once with staining buffer (PBS containing 3\% FCS and 2 mM EDTA) and the cells were stained in $100 \mu \mathrm{l}$ staining buffer for $20 \mathrm{~min}$ at room temperature in the dark. For enumeration of lamina propria dendritic cells, directly labeled monoclonal antibodies for the following markers were used: lin (lineage) 1-FITC (CD3, CD14, CD16, CD19, CD20, CD56, and CD34), HLA-DR-PerCP-Cy5.5, CD11c-APC, and CD103PE. LPDCs were identified as lin1-/HLA-DR ${ }^{+}$cells. For determination of Tregs, anti-CD3-APC-Cy7, anti-CD4V450, anti-CD8-FITC, anti-CD25-PE, and anti-CD127Alexa Fluor 647 antibodies were used. With the exception of CD103-PE (eBioscience, San Diego, USA), all antibodies were purchased from BD Bioscience (San Jose, USA). FMO (fluorescence-minus-one) controls were employed to set the boundaries for gating of positively stained cells. After the staining reaction, the cells were washed once with staining buffer and re-suspended in $100 \mu \mathrm{l}$ staining buffer. For the exclusion of dead cells, propidium iodide (PI) was added to the samples immediately prior to acquisition on an LSR II (BD Bioscience, San Jose, USA) flow cytometer. The data files were analyzed using FlowJo (FlowJo, LLC) software.

\section{Isolation of total genomics DNA, 16 S library preparation, and Illumina sequencing}

Stool and mucosal samples were stored at $-80^{\circ} \mathrm{C}$ and used for total DNA isolation combining mechanical and enzymatic lysis with the MagnaPure LC DNA Isolation Kit III (Bacteria, Fungi) (Roche, Mannheim, Germany) according to manufacturer's instructions as described [30]. Modifications were made for stool and mucosal specimens. Briefly, stool samples were homogenized in $500 \mu \mathrm{l}$ PBS and $250 \mu \mathrm{l}$ of the suspension was mixed with $250 \mu \mathrm{l}$ of bacterial lysis buffer and further transferred to Magna Lyser green bead tubes (Roche, Mannheim, Germany). Mechanical lysis was two times performed at $6500 \mathrm{rpm}$ in a MagNA Lyser Instrument (Roche, Mannheim, Germany). Mucosal specimens were prepared with bead beating for four times at $6500 \mathrm{rpm}$ in $500 \mu \mathrm{l}$ lysis buffer and enzymatic lysis samples were mixed with $25 \mu \mathrm{l}$ lysozyme $(100 \mathrm{mg} / \mathrm{ml})$ and incubated at $37{ }^{\circ} \mathrm{C}$ for $30 \mathrm{~min}$. Afterwards, samples were mixed with $30 \mu$ l Proteinase $\mathrm{K}$ and stool samples were incubated at $65^{\circ} \mathrm{C}$ for $1 \mathrm{~h}$. Mucosal specimens were incubated overnight at $65^{\circ} \mathrm{C}$. Enzymes were heat inactivated at $95^{\circ} \mathrm{C}$ for $10 \mathrm{~min}$ and further steps were performed according to Magna Pure DNA isolation kit III (Bacteria, Fungi) manufacturer's instruction. $250 \mu \mathrm{l}$ of mucosal specimens and $100 \mu \mathrm{l}$ of stool samples were taken for DNA purification that was eluted in $100 \mu \mathrm{l}$. For target specific PCR amplification, the primers 
27f (AGAGTTTGATCCTGGCTCAG) and 357r (CTGCTG CCTYCCGTA) were taken as described by Baker et al. [31] and synthesized at Eurofins (MWG, Ebersberg, Germany). Then, $5 \mu$ of total DNA from mucosal sample and $2 \mu$ from stool sample extracts were taken for a $25 \mu \mathrm{l}$ PCR reactions as described [30]. Triplicates were pooled and amplification was verified using a $1 \%$ agarose gel. The sequencing library was amplified, quantified, and sequenced on a MiSeqII desktop sequencer (Illumina, Eindhoven, The Netherlands) as described previously [30]. Version 3.600 cycles chemistry (Illumina, Eindhoven, The Netherlands) was taken according to manufacturer's instructions to run the $6 \mathrm{pM}$ library with 20\% PhiX (Illumina, Eindhoven, The Netherlands). FASTQ files were then further taken to perform data analysis.

\section{Microbiota analysis and statistical methods}

Quality filtering and analysis of raw 16S rRNA gene sequence data (hypervariable region V1-V2) was performed with mothur (version 1.22.0) according to the recommended standard operating procedure of mothur for Illumina MiSeq data (https://www.mothur.org/wiki/MiSeq_SOP, accessed June 2016) with additional removal of singletons (default settings and parameters were used, if not specified otherwise) [32, 33]. Briefly: paired reads were merged using mothur's make.contigs command, whereby reads less than 200 bps were filtered out of the data set. In addition, sequences containing ambiguous bases or more than eight homopolymeres were removed together with chimeric sequences or sequences outside of the core alignment with the SILVA reverence database (version 119). Furthermore, noisy sequences were identified using pre.cluster and finally deleted from the data set. Remaining pre-processed and filtered sequences were clustered by mothur's de novo OTUpicking strategy into OTUs at a distance of 0.03 . Finally, taxonomic classification was assigned using the RDP Bayesian classifier (version 2.10.1, trainingsset 10/29.10.2014) with default settings and a classification confidence cutoff of $80 \%$ [34]. Subsequent OTU-based microbiota analyses were performed in QIIME (version 1.8.0), including core. diversity analysis with rarefaction to a sampling depth of 9.538 reads per sample for all four locations $(\mathrm{COR}=$ corpus, $\mathrm{COL}=$ colon, $\mathrm{FEC}=$ feces, and $\mathrm{DUO}=$ duodenum) [35]. Unweighted UniFrac distance metrics as measure of between-sample (beta) diversity was calculated and applied for principal coordinates analysis (PCoA) to visualize patterns of diversity [36]. Within-samples (alpha) diversity was calculated using four different measures (1) observed species, (2) ChaoI Index, (3) Shannon Index, and (4) Faith's phylogenetic diversity $[37,38]$. Statistical significant differences between sample (alpha) diversity were assessed by a nonparametric two-sample $t$ test ( $p$ values were determined by Monte Carlo permutations. Calculations are based on the greatest rarefaction depth. Bonferroni correction was used to account for multiple comparisons). Differences in taxonomic microbiota compositions (differentially abundant features/ genera) within the four locations and between treatments were determined using linear discriminant effect size analysis (LEfSe) on the filtered data sets at species level. If not otherwise specified $p$ values below 0.05 were considered as statistically significant [38].

\section{Availability of data and materials}

The sequence data supporting the results of this article are available in the European Bioinformatics Institute Sequence Read Archive under accession number PRJEB19253.

\section{Extract preparation from specimens for multiplex cytokine assay}

Colonic and duodenum specimens were obtained in cold RPMI1640 medium supplemented with penicillin and streptomycin. Samples were transferred to cryotubes, snap frozen, and stored in liquid nitrogen until sample preparation. All samples were then individually thawed on ice and immediately disrupted in $300 \mu \mathrm{l}$ extraction buffer for 2 min on ice with a pellet pestle (Kimble Kontes, USA). Extraction buffer comprised DPBS (Dulbecco's phosphate buffered saline without calcium and magnesium, Lonza) and EDTAfree protease inhibitors (cOmplete mini, Roche). After further disrupting them mechanically by pipetting, biopsies were passed through a $70 \mu \mathrm{m}$ cell strainer. All samples were then incubated on ice for $5 \mathrm{~min}$. Finally, supernatants were obtained by centrifugation at $10,000 \times g$ for $10 \mathrm{~min}$ at $4{ }^{\circ} \mathrm{C}$, snap frozen in liquid nitrogen, and stored at $-80{ }^{\circ} \mathrm{C}$ until analysis. Cytokine analysis included IL- $1 \beta$, IL-6, IL-10, IL-12p40, IL-12p70, IL-17A, IL-23, and TNF- $\alpha$. Multiplex immunoassay kits (ProcartaPlex) used for analysis were obtained from eBioscience and were run according to manufacturer's instructions using magnetic beads. Standards for each cytokine were assayed in duplicates to generate standard curves using the reference concentrations as provided by the manufacturer. All samples were individually thawed on ice and wash steps were performed using a hand-held magnetic block. Data were obtained on a validated and calibrated Bio-Plex 200 system (Bio-Rad) and analyzed with BioPlex Manager 6.1 software (Bio-Rad). BCA Protein Assay (Pierce) was used to determine total protein concentration and to normalize cytokine concentrations for each sample.

\section{GC-EI/MS of short-chain fatty acids}

SCFAs (acetic acid, propionic acid, iso-butyric acid, butyric acid, iso-valeric acid, and valeric acid) were extracted from stool frozen at $-80{ }^{\circ} \mathrm{C}$. SCFA concentrations were 
measured by a GC-MS equipped with a PEG DB-WAXetr. ( $30 \mathrm{~m} ; 0.25 \mathrm{~mm}$ ID; and $0.25 \mu \mathrm{m}$ film) column. SCFA were extracted from feces by sequential addition of $1 \mathrm{ml}$ phosphoric acid (0.5\%) and $1 \mathrm{ml}$ methyl-tert-butyl-ether, $10 \mathrm{~min}$ shaking, 10 min centrifugation, and removal of the upper organic layer. Before extraction $100 \mathrm{nmol}$ of D-acetic acid, D-propionic acid, D-butyric acid, and D-valeric acid were added as internal standards. Calibration curves by stable isotope dilution were performed from 0.1 to $2.000 \mu \mathrm{M}$ for acetic acid, propionic acid, iso-butyric acid, butyric acid, iso-valeric acid, and valeric acid. A 7890B/5977A MSD GC-MS (Agilent, Waldbronn, Germany) equipped with a PEG DB-WAXetr. (30 m; $0.25 \mathrm{~mm}$ ID; and $0.25 \mu \mathrm{m}$ film) column was used. Helium was used as carrier gas at $1.3 \mathrm{ml} / \mathrm{min}$ in splitless mode at $250{ }^{\circ} \mathrm{C}$ injector temperature. The initial oven temperature of $60^{\circ} \mathrm{C}$ was held for $2 \mathrm{~min}$, and then, the temperature first was ramped up to $150^{\circ} \mathrm{C}$ at a rate of $15^{\circ} \mathrm{C} / \mathrm{min}$. This was followed by a ramp of $5^{\circ} \mathrm{C} / \mathrm{min}$ up to $170^{\circ} \mathrm{C}$ and $20^{\circ} \mathrm{C} / \mathrm{min}$ up to $250^{\circ} \mathrm{C}$, where the temperature was held for another $2 \mathrm{~min}$. The mass spectrometer was run in electron impact (EI) mode, where the SCFAs were detected in SIM mode on $\mathrm{m} / \mathrm{z} 60,63,73,74,76,79$, and 80. The source temperature was set to $250{ }^{\circ} \mathrm{C}$ and the transfer line temperature was $280^{\circ} \mathrm{C}$. Data analysis was performed by Mass Hunter (Agilent, Waldbronn, Germany).

\section{Zonulin}

A ready-to-use solid-phase sandwich ELISA (Immundiagnostik AG, Bensheim, Germany) was used to detect zonulin (zonulin Stool ELISA) in fecal samples. The tests were performed according to the manufacturer's instructions. For stool sampling, the Stool Sample Application System (Immundiagnostik AG, Bensheim, Germany) was used according to the manufacturer's manual [39].

\section{Statistics}

Statistical analyses were carried out using SPSS $22\left(\right.$ IBM $^{\circledR}$ Corporation USA) and GraphPad Prism ${ }^{\circledR} 6.0$ (GraphPad Software, Inc., USA). Values are presented as number (\%) or median [interquartile range]. For the comparison of categorical variables, we applied Fisher's exact test. Group differences of continuous variables were determined by Mann-Whitney $U$ test or $t$ test depending on the data distribution (non-gaussian or gaussian). Boxplots are depicted according to Tukey.

\section{Results}

\section{Patients}

Ten patients with IBS-D consented to the study. Their median age was 46 [37-53] years (median [Q1-Q3]) of which 5/10 were women. Four patients had no relevant medical comorbidities, and those of the remaining six patients are compiled in Table 1.

\section{Effect of synbiotic treatment on mucosal immune cell lineages and mucosal cytokine levels in different regions of the gastrointestinal tract}

We performed FACS analyses of human mucosal specimens (biopsies from the duodenum, ascending colon) after collection of tissue samples during endoscopy before and after synbiotic treatment. Mucosal T- and dendritic cell subsets were then characterized by FACS analysis. Mucosal immune cell results are systematically listed in suppl. Table 1 . In the ascending colon, a significant reduction of mucosal $\mathrm{CD} 4^{+} \mathrm{T}$ cells (60 [57-65] vs. 55 [50-60] \%, $p=0.042)$ was observed after synbiotic treatment (Fig. 1). Furthermore, double-negative $\mathrm{T}$ cells $\left(\mathrm{CD}^{-}{ }^{-} \mathrm{CD} 4^{-} \mathrm{T}\right.$ cells) showed a trend towards elevation (10 [9-12] vs. 13 [8-25] \%, $p=0.078)$ after synbiotic treatment in the ascending colon. Dendritic cells could not be isolated from the duodenal mucosa. No synbiotic treatment-associated changes of DCs (total, CD11 $\mathrm{c}^{+}$, or $\mathrm{CD}_{103^{+}}$) were found in the ascending colon. No significant changes of T-cell subsets were found in the duodenum.

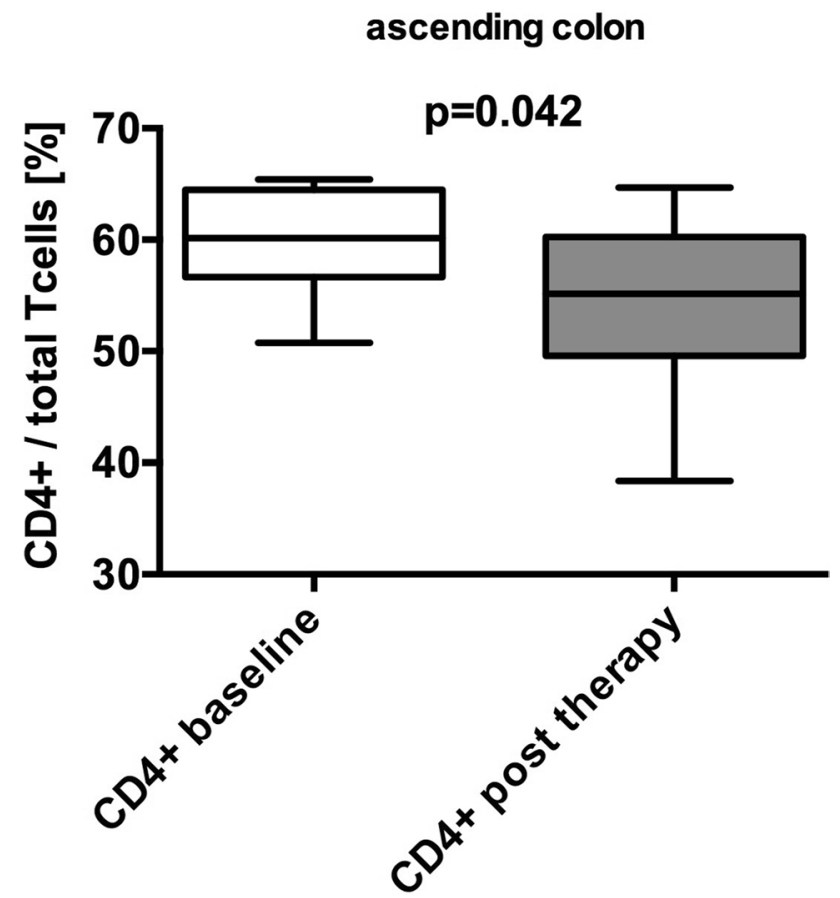

Fig. 1 Reduction of mucosal $\mathrm{CD} 4^{+} \mathrm{T}$ cells after synbiotic treatment. A significant reduction of $\mathrm{CD}^{+} \mathrm{T}$ cells $(\%)$, sampled from mucosa specimens from the ascending colon, was found after (grey) synbiotic treatment compared to baseline (white) (60 [57-65] vs. 55 [50-60], $p=0.042$ ) (median [Q1-Q3]; Mann-Whitney $U$ test or $t$ test to compare non-gaussian and gaussian variables. Boxplots according to Tukey) 
Mucosal extract cytokine concentration was measured in mucosal specimens of the duodenum and ascending colon and results are systematically compiled in Suppl. Table 2. A trend towards higher concentration of tumor necrosis factor alpha (TNF-alpha) was evident after synbiotic treatment in the ascending colon $(0.2[0-0.4]$ vs. $0.6[0-1.1] \mathrm{pg} / \mathrm{ml}$, $p=0.0547)$. Other cytokines were not affected by synbiotic treatment.

\section{Increased phylogenetic diversity in the upper but not lower gastrointestinal tract after synbiotic treatment}

To further elucidate the effects of synbiotic treatment on different regions of the gastrointestinal tract (gastric corpus, duodenum, ascending colon, feces), richness (observed species) phylogenetic diversity (Faith's phylogenetic diversity), and Shannon Diversity Index in mucosal and fecal samples were determined. Significant elevations in phylogenetic diversity could be observed in mucosal samples of the gastric corpus $(p=0.008)$ and duodenum $(p=0.025)$ as well as richness in the duodenum $(p=0.011)$. No differences were found in colonic $(p=0.710)$ and fecal samples $(p=0.358$, Fig. 2). All results are shown in suppl. Table 3.

\section{Microbial abundances associated to synbiotic treatment}

Bacterial community profiling was performed to investigate upper and lower gastrointestinal mucosal specimens and fecal samples. Thereby, mucosal specimens of the gastric corpus revealed raised relative abundances of unclassified Halomonas ( $p=0.007)$, unclassified Neisseriaceae ( $p=0.010)$, Propionibacterium acnes $(p=0.040)$, and Clostridiaceae $(p=0.029)$, whereas Actinobacteria $(p=0.027)$ were reduced after synbiotic treatment. In duodenal specimens, elevated unclassified Schwartzia $(p=0.013)$ and Catonella $(p=0.029)$ in addition to a reduction of an unclassified Lactobacillus after synbiotic treatment was evident. No taxa showing significantly different relative abundances before and after treatment were observed in colonic mucosal specimens. No probiotic strain of the synbiotic mixture could colonize the gut. A higher abundance of unclassified Lactobacillaceae $(p=0.006)$ could be found in fecal samples after synbiotic treatment. Moraxella $(p=0.022)$ and Moryella $(p=0.022)$ were reduced after treatment in fecal samples (Fig. 3).
Fig. 2 Increased phylogenetic diversity in the upper- but not lower gastrointestinal tract after synbiotic treatment. a, b Phylogenetic diversity (Faith) increased in specimens from the gastric corpus $(p=0.008)$ and duodenum $(p=0.025)$. $\mathbf{c}$, d No differences in colonic samples $(p=0.710)$ and feces $(p=0.358)$ were evident (Faith's phylogenetic diversity, based on UniFrac phylogenetic distance, nonparametric two-sample $t$ test to determine $p$ values using Monte Carlo permutations, post-error correction Bonferroni)
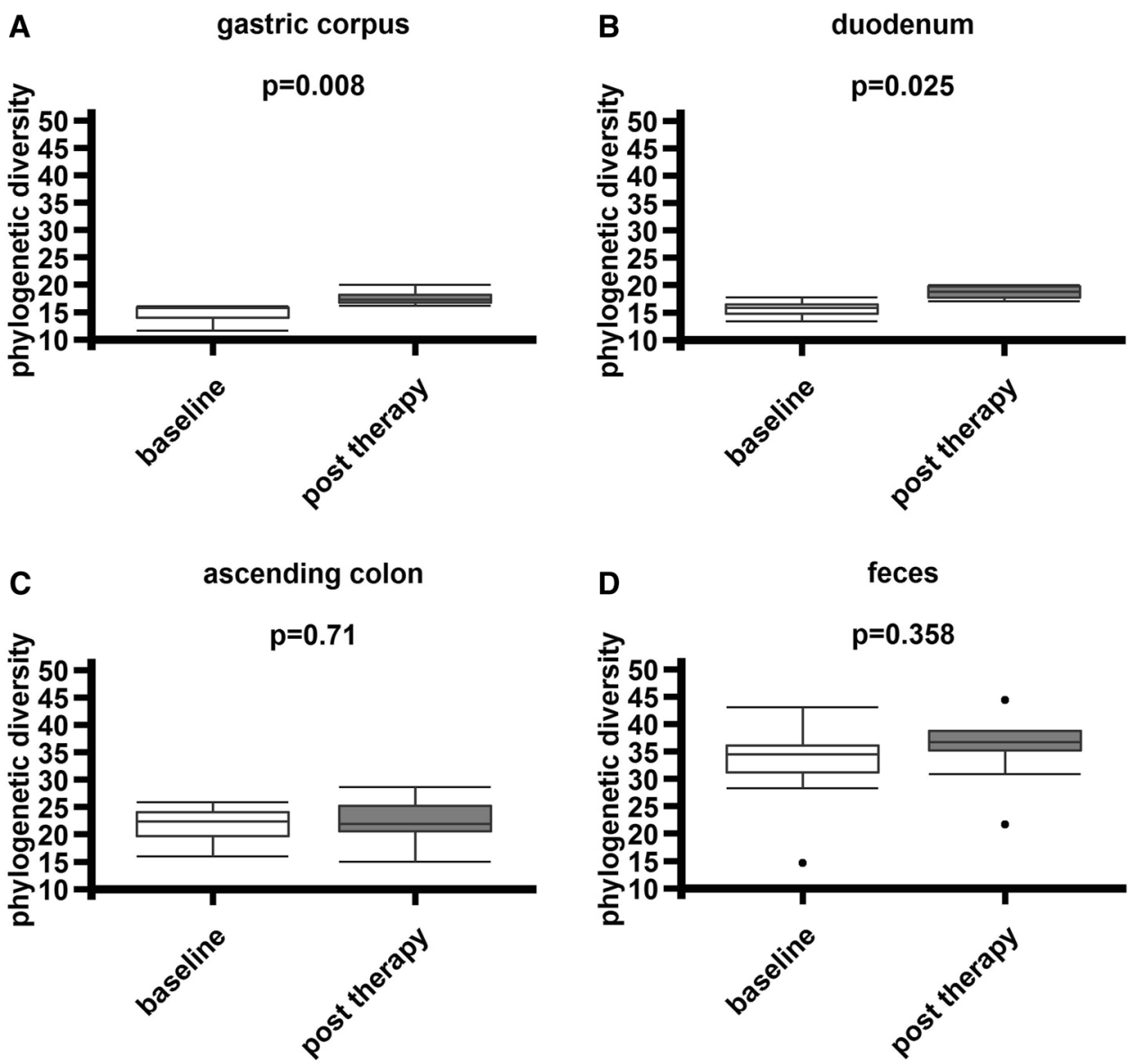
A

more abundant after treatment

more abundant before treatment

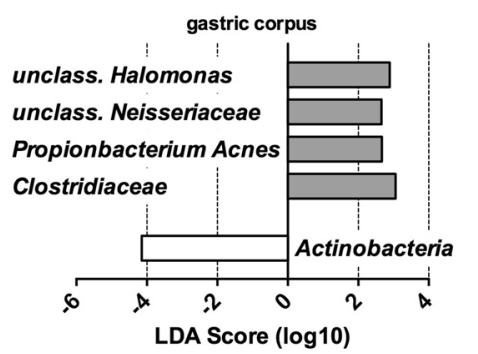

C
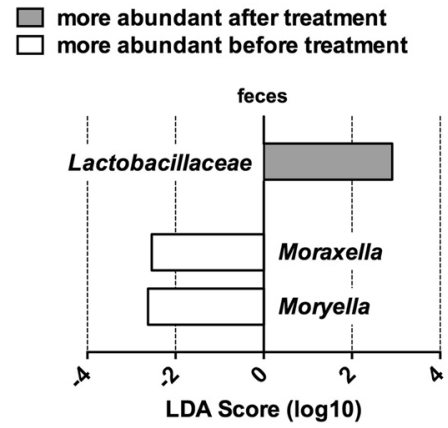

B

more abundant after treatment

more abundant before treatment

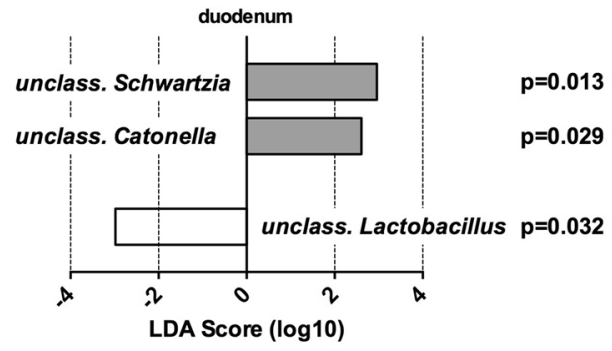

$p=0.006$

$p=0.022$

$p=0.022$
Fig. 3 Linear discriminant analysis of mucosal and fecal samples pre- and post-synbiotic treatment. Linear discriminant analysis (LDA) was generated with LEfSe. a Mucosal specimens of the gastric corpus showed elevated relative abundances of unclassified Halomonas $(p=0.007)$, unclassified Neisseriaceae $(p=0.010)$, Propionibacterium Acnes $(p=0.040)$, and Clostridiaceae $(p=0.029)$ together with a reduction of Actinobacteria $(p=0.027)$ after synbiotic treatment. $\mathbf{b}$

\section{Increased SCFA levels and reduction of fecal zonulin in fecal samples after synbiotic treatment}

We examined levels of SCFAs in the fecal samples of patients by high-performance liquid chromatography (HPLC). Significant increases in acetate (101 [79-133] vs. 202 [68-252] $\mu \mathrm{mol} / \mathrm{g} ; p=0.007)$ and butyrate $(27$ [14-35] vs. 40 [25-67] $\mu \mathrm{mol} / \mathrm{g} ; p=0.037$ ) levels after treatment were found (Fig. 4), whereas propionate, iso-butyrate, iso-valerate, and valerate levels remained unaltered. Fecal concentrations of zonulin were measured using competitive ELISA in fecal samples. Zonulin concentrations $(\mathrm{ng} / \mathrm{ml})$ decreased significantly after synbiotic treatment (67 [38-92] vs. 36 [20-48] ng/ml; $p=0.035$; Suppl. Figure 1).

\section{Reduction of the symptom severity after synbiotic treatment}

The IBS-SSS (irritable bowel syndrome-severity scoring system) was used to define clinical effects of synbiotic treatment. Patients showed significant improvements in IBS-SSS. (237 [129-256] vs. 54 [12-158]; $p=0.002$; Suppl. Figure 2).
Mucosal specimens of the duodenum depicted increased unclassified Schwartzia $(p=0.013)$ and Catonella $(p=0.029)$ as well as a reduction of an unclassified Lactobacillus after synbiotic treatment. $\mathbf{c}$ Fecal samples showed increased Lactobacillaceae $(p=0.006)$ as well as diminished Moraxella $(p=0.022)$ and Moryella $(p=0.022)$ after synbiotic treatment

\section{Discussion}

In the present pilot study, we conducted a systematic investigation of the GI tract before and after a 4-week treatment with an open-label oral synbiotic mixture in patients with IBS-D. We thereby assessed the mucosal immune system and microbiota, fecal microbiota and SCFAs, clinical IBS activity, and mucosal permeability. Our data suggest varying GI effects of oral synbiotic treatment in IBS-D patients. The most significant changes were seen in mucosal microbiota biodiversity of the upper GI tract, accompanied with effects on mucosal immune cell subsets, microbial metabolic activity and small intestinal mucosal barrier function reflected by zonulin levels [40].

First, synbiotic treatment influenced mucosal phylogenetic bacterial diversity in the upper but not the lower gastrointestinal tract showing pronounced increases of phylogenetic diversity of the microbiota in gastric and duodenal mucosa, whereas diversity remained unaffected in colon and feces. In addition, Lactobacillaceae, belonging to the synbiotic mixture, were elevated only in fecal samples, but were not found in any mucosal sample. Fecal samples depicted 
Fig. 4 Elevated SCFA levels in fecal samples of patients after synbiotic treatment. Fecal samples were analyzed by HPLC. A significant elevation in fecal acetate and butyrate levels ( $\mu \mathrm{mol} / \mathrm{g}$ ) before (white) vs. after synbiotic treatment (grey) were found. Acetate (101 [79-133] vs. 202 [68-252]; $p=0.007)$ and butyrate content $(27[14-35]$ vs. 40 [25-67]; $p=0.037$ ) (median [Q1-Q3]; Mann-Whitney $U$ test or $t$ test to compare nongaussian and gaussian variables. Boxplots according to Tukey)
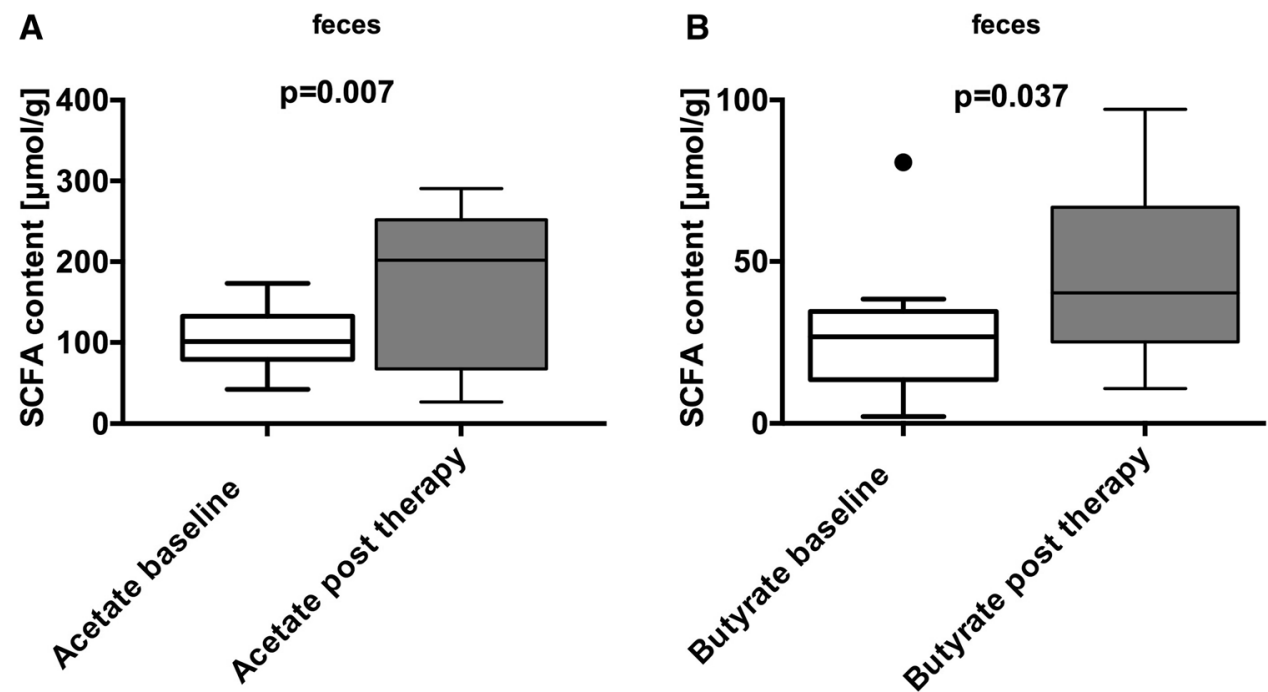

a notably reduction of Moraxella and Moryella, but the importance of this observations is unclear. The observed elevations of phylogenetic diversity in gastric and duodenal specimens were not chaperoned by increases of the orally administered probiotic strains used for treatment, which is a known phenomenon in probiotic therapy. Nevertheless, oral probiotics might have a catalyzing effect on mucosal richness [13]. Although changes in microbial composition of the rectal mucosa have been described after probiotic therapy of IBS patients, we herein show a more comprehensive investigation of how synbiotic preparation affects the intestinal ecosystem in different regions of the GI tract [19]. Furthermore, our results suggest that synbiotic effects on microbial composition in IBS-D are preferentially observed in the upper gastrointestinal tract. Whether the observed treatment-induced changes are due to the probiotic or prebiotic ingredients of the administered mixture remain unclear, since prebiotics are also known to influence intestinal microbial composition in humans [41]. Taken together, this study gives a comprehensive oversight of $16 \mathrm{~S}$ rRNA changes in different habitats and locations of the gastrointestinal tract induced by an oral synbiotic. Specifically, we show for the first time that the focus of mucosal microbial responses to oral synbiotic therapy is located in the upper but not the lower gastrointestinal tract.

Second, oral synbiotic treatment significantly reduced the number of $\mathrm{CD}^{+} \mathrm{T}$ cells in the ascending colon. Increased mucosal inflammation is associated with IBS-D pathogenesis and disease activity. Moreover, elevated numbers of $\mathrm{T}$ cells have been shown in jejunal and colonic specimen of IBS-D patients vs. controls. However, these cells were not differentiated further, which could be of relevance, because naïve $\mathrm{T}$ cells can develop into pro-as well as anti-inflammatory $\mathrm{T}$ cells $[5,42,43]$. The treatment-induced reduction of colonic $\mathrm{CD}^{+}{ }^{+} \mathrm{T}$ cells in our study is likely to lead to an alteration of the colonic inflammatory status. In conjunction with the observed trend towards an induction of double-negative $\left(\mathrm{CD}^{-} \mathrm{CD}^{-}\right) \mathrm{T}$ cells, for which Treg-like anti-inflammatory properties (suppression of $\mathrm{CD}^{+}$and $\mathrm{CD} 4^{+} \mathrm{T}$ cells, $B$ cells and DCs) have been proposed, one could hypothesize an anti-inflammatory effect [44]. It is one of the limitations of this study that we neither differentiated further $\mathrm{CD}^{+} \mathrm{T}$ cells into pro- and anti-inflammatory subsets. It is worth noting that $\mathrm{T}$ cells in the upper GI tract, where the microbial changes were most pronounced, remained unaffected. In addition, neither mucosal regulatory $\mathrm{T}$ cells nor DCs could be shown to be affected by synbiotics in any compartment. In sum, the reduction of mucosal $\mathrm{CD}^{+} \mathrm{T}$ cells accompanied by enhanced double-negative $\mathrm{T}$ cells in the ascending colon could represent an anti-inflammatory modulation of the colonic mucosa, but this needs further confirmation.

Third, we show that synbiotic treatment elevated fecal levels of the SCFAs acetate and butyrate. The synbiotic mixture could stimulate acetate and butyrate levels by its prebiotic or probiotic components, as prebiotics are known to stimulate, e.g., butyrate production in humans and Lactobacillaceae (elevated in fecal samples after treatment) can mediate butyrate production [41, 45]. Furthermore, probiotic bacteria are metabolically active depending on the host microbiota and initial fecal butyrate level in healthy individuals. Thereby, the impact of probiotics on fecal butyrate level was highest in individuals with low butyrate concentration, as observed in our study participants, before treatment [18, 22, 46]. It was previously demonstrated that IBS patients show decreased fecal butyrate levels, which is of importance, since acetate and butyrate are known to induce mucosal Tregs and regulatory DCs [22, 47]. We hypothesize that the treatment-associated elevation of acetate and butyrate could be involved in the observed mucosal T-cell changes and the clinical responses to the study preparation. 
Intestinal SCFAs such as butyrate are also known to stabilize intestinal barrier function even after chemical disruption and might, therefore, be of importance in prophylaxis and treatment of gut leakiness [48, 49]. IBS-D patients exert intestinal permeability disruptions and abnormalities in their epithelial barrier function, as shown in the previous studies $[8,10]$. In this study, fecal zonulin concentration, a surrogate marker for the intestinal barrier, was significantly reduced by synbiotics. This reflects a possible treatment effect on intestinal barrier function in our IBS-D cohort. The stabilization of the epithelial barrier could subsequently influence mucosal inflammation in IBS-D $[9,10]$ and contribute to a significantly lower symptom severity score after treatment. In sum, synbiotic therapy elevated fecal acetate and butyrate levels accompanied by a reduction of fecal zonulin and symptom severity. These observations indicate possible effects of synbiotic therapy on microbiota metabolism and intestinal barrier function.

In conclusion, the present pilot study indicates possible mechanisms of action of oral synbiotic therapy in IBS-D. The observed effects comprise an elevation of mucosal microbial diversity, the elevation of colonic $\mathrm{CD} 4^{+} \mathrm{T}$ cells, the elevation of fecal acetate and butyrate levels and a decrease of fecal zonulin, and a surrogate of intestinal barrier function. The potency of oral synbiotics in the treatment of IBS-D is underlined by the clinical response observed in this study by decreased IBS-SSS counts. The major limitations of the study are the lack of a placebocontrolled double-blinded design and the limited number of participants, which are limitations owing to the design as a pilot study. This in turn does not allow any causal interpretation of the results. Nevertheless, our study provides a systematic analysis of the possible effects of oral synbiotic therapy in IBS-D patients and gives the rationale for a larger-scale randomized trial.

Acknowledgements Open access funding provided by Medical University of Graz. We thank Jennifer Ober for her efforts in performing FACS analyses and Harald Köfeler for his support regarding SCFA measurements.

Author contributions AMM and WS planned the study and experiments, analyzed data, and wrote the paper. $\mathrm{CH}$ supervised study planning and implementation and gave scientific advices. AMM, PK, and $\mathrm{CH}$ recruited patients. WS, $\mathrm{PK}$, and $\mathrm{CH}$ performed endoscopies. HS performed immunological analyses. GG and BH performed microbiota analysis and microbiota statistics. All authors were involved in the preparation of the manuscript and data discussion.

Funding Institut Allergosan GmbH, Graz, Austria sponsored synbiotics, biometric measurements and the costs for study coordination. Institute Allergosan $\mathrm{GmbH}$ did not have influence on study design and approval, patient recruitment, measurements, and data analysis or interpretation.

\section{Compliance with ethical standards}

Ethics approval and consent to participate All protocols and informed consents were a priori waived by the institutional review board (IRB number IRB00002556) of the Medical University of Graz, vote 25-594 ex 12/13. All individuals included signed an informed consent prior to study inclusion.

Prior publication as part of dissertation The data used in this publication are part of the Dissertation of Adrian M. Moser.

Consent for publication Not applicable.

Conflict of interest A. M. M. was employed at the Medical University of Graz for study coordination by a scientific grant from Institute Allergosan GmbH. A. M. M, C. H., and P. K. received payment from Institute Allergosan $\mathrm{GmbH}$ as speaker. All the other authors declare not to have any competing interests with regard to the current study.

Open Access This article is distributed under the terms of the Creative Commons Attribution 4.0 International License (http://creativeco mmons.org/licenses/by/4.0/), which permits unrestricted use, distribution, and reproduction in any medium, provided you give appropriate credit to the original author(s) and the source, provide a link to the Creative Commons license, and indicate if changes were made.

\section{References}

1. Lovell RM, Ford AC (2012) Global prevalence of and risk factors for irritable bowel syndrome: a meta-analysis. Clin Gastroenterol Hepatol 10(7):712 e714-721 e714. https://doi.org/10.1016/j. cgh.2012.02.029

2. Andresen V, Keller J, Pehl C, Schemann M, Preiss J, Layer P (2011) Irritable bowel syndrome-the main recommendations. Dtsch Arztebl Int 108(44):751-760. https://doi.org/10.3238/arzte b1.2011.0751

3. Enck P, Aziz Q, Barbara G, Farmer AD, Fukudo S, Mayer EA, Niesler B, Quigley EM, Rajilic-Stojanovic M, Schemann M, Schwille-Kiuntke J, Simren M, Zipfel S, Spiller RC (2016) Irritable bowel syndrome. Nat Rev Dis Primers 2:16014. https://doi. org/10.1038/nrdp.2016.14

4. Hughes PA, Harrington AM, Castro J, Liebregts T, Adam B, Grasby DJ, Isaacs NJ, Maldeniya L, Martin CM, Persson J, Andrews JM, Holtmann G, Blackshaw LA, Brierley SM (2013) Sensory neuro-immune interactions differ between irritable bowel syndrome subtypes. Gut 62(10):1456-1465. https://doi. org/10.1136/gutjnl-2011-301856

5. Guilarte M, Santos J, de Torres I, Alonso C, Vicario M, Ramos L, Martinez C, Casellas F, Saperas E, Malagelada JR (2007) Diarrhoea-predominant IBS patients show mast cell activation and hyperplasia in the jejunum. Gut 56(2):203-209. https://doi. org/10.1136/gut.2006.100594

6. Liu Y, Zhang L, Wang X, Wang Z, Zhang J, Jiang R, Wang X, Wang K, Liu Z, Xia Z, Xu Z, Nie Y, Lv X, Wu X, Zhu H, Duan L (2016) Similar fecal microbiota signatures in patients with diarrhea-predominant irritable bowel syndrome and patients with depression. Clin Gastroenterol Hepatol 14(11):1602 e1605-1611 e1605. https://doi.org/10.1016/j.cgh.2016.05.033

7. Rigsbee L, Agans R, Shankar V, Kenche H, Khamis HJ, Michail S, Paliy O (2012) Quantitative profiling of gut microbiota of children with diarrhea-predominant irritable bowel syndrome. Am 
J Gastroenterol 107(11):1740-1751. https://doi.org/10.1038/ ajg.2012.287

8. Martinez C, Rodino-Janeiro BK, Lobo B, Stanifer ML, Klaus B, Granzow M, Gonzalez-Castro AM, Salvo-Romero E, AlonsoCotoner C, Pigrau M, Roeth R, Rappold G, Huber W, Gonzalez-Silos R, Lorenzo J, de Torres I, Azpiroz F, Boulant S, Vicario M, Niesler B, Santos J (2017) miR-16 and miR-125b are involved in barrier function dysregulation through the modulation of claudin-2 and cingulin expression in the jejunum in IBS with diarrhoea. Gut. https://doi.org/10.1136/gutjnl-2016-31147 7

9. Vicario M, Gonzalez-Castro AM, Martinez C, Lobo B, Pigrau M, Guilarte M, de Torres I, Mosquera JL, Fortea M, Sevillano-Aguilera C, Salvo-Romero E, Alonso C, Rodino-Janeiro BK, Soderholm JD, Azpiroz F, Santos J (2015) Increased humoral immunity in the jejunum of diarrhoea-predominant irritable bowel syndrome associated with clinical manifestations. Gut 64(9):1379-1388. https://doi.org/10.1136/gutjnl-2013-306236

10. Martinez C, Lobo B, Pigrau M, Ramos L, Gonzalez-Castro AM, Alonso C, Guilarte M, Guila M, de Torres I, Azpiroz F, Santos J, Vicario M (2013) Diarrhoea-predominant irritable bowel syndrome: an organic disorder with structural abnormalities in the jejunal epithelial barrier. Gut 62(8):1160-1168. https://doi. org/10.1136/gutjnl-2012-302093

11. Moayyedi P, Ford AC, Talley NJ, Cremonini F, Foxx-Orenstein AE, Brandt LJ, Quigley EM (2010) The efficacy of probiotics in the treatment of irritable bowel syndrome: a systematic review. Gut 59(3):325-332. https://doi.org/10.1136/gut.2008.167270

12. Klaenhammer TR, Kleerebezem M, Kopp MV, Rescigno M (2012) The impact of probiotics and prebiotics on the immune system. Nat Rev Immunol 12(10):728-734. https://doi.org/10.1038/ nri3312

13. Gerritsen J, Smidt H, Rijkers GT, de Vos WM (2011) Intestinal microbiota in human health and disease: the impact of probiotics. Genes Nutr 6(3):209-240. https://doi.org/10.1007/s1226 3-011-0229-7

14. Didari T, Mozaffari S, Nikfar S, Abdollahi M (2015) Effectiveness of probiotics in irritable bowel syndrome: updated systematic review with meta-analysis. World J Gastroenterol 21(10):30723084. https://doi.org/10.3748/wjg.v21.i10.3072

15. Rossi O, van Berkel LA, Chain F, Tanweer Khan M, Taverne N, Sokol H, Duncan SH, Flint HJ, Harmsen HJ, Langella P, Samsom JN, Wells JM (2016) Faecalibacterium prausnitzii A2-165 has a high capacity to induce IL-10 in human and murine dendritic cells and modulates T cell responses. Sci Rep 6:18507. https://doi. org/10.1038/srep18507

16. Atarashi K, Tanoue T, Oshima K, Suda W, Nagano Y, Nishikawa H, Fukuda S, Saito T, Narushima S, Hase K, Kim S, Fritz JV, Wilmes P, Ueha S, Matsushima K, Ohno H, Olle B, Sakaguchi S, Taniguchi T, Morita H, Hattori M, Honda K (2013) Treg induction by a rationally selected mixture of Clostridia strains from the human microbiota. Nature 500(7461):232-236. https://doi. org/10.1038/nature12331

17. Mao X, Gu C, Hu H, Tang J, Chen D, Yu B, He J, Yu J, Luo J, Tian G (2016) Dietary Lactobacillus rhamnosus GG supplementation improves the mucosal barrier function in the intestine of weaned piglets challenged by porcine rotavirus. PLoS One 11(1):e0146312. https://doi.org/10.1371/journal.pone.0146312

18. Gargari G, Taverniti V, Balzaretti S, Ferrario C, Gardana C, Simonetti P, Guglielmetti S (2016) Consumption of a Bifidobacterium bifidum strain for 4 weeks modulates dominant intestinal bacterial taxa and fecal butyrate in healthy adults. Appl Environ Microbiol 82(19):5850-5859. https://doi.org/10.1128/ AEM.01753-16

19. Ng SC, Lam EF, Lam TT, Chan Y, Law W, Tse PC, Kamm MA, Sung JJ, Chan FK, Wu JC (2013) Effect of probiotic bacteria on the intestinal microbiota in irritable bowel syndrome. J Gastroenterol Hepatol 28(10):1624-1631. https://doi.org/10.1111/ jgh. 12306

20. Zeng J, Li YQ, Zuo XL, Zhen YB, Yang J, Liu CH (2008) Clinical trial: effect of active lactic acid bacteria on mucosal barrier function in patients with diarrhoea-predominant irritable bowel syndrome. Aliment Pharmacol Ther 28(8):994-1002. https://doi. org/10.1111/j.1365-2036.2008.03818.x

21. Lee BJ, Bak YT (2011) Irritable bowel syndrome, gut microbiota and probiotics. J Neurogastroenterol Motil 17(3):252-266. https ://doi.org/10.5056/jnm.2011.17.3.252

22. Farup PG, Rudi K, Hestad K (2016) Faecal short-chain fatty acids-a diagnostic biomarker for irritable bowel syndrome? BMC Gastroenterol 16(1):51. https://doi.org/10.1186/s1287 6-016-0446-Z

23. IFord AC, Quigley EM, Lacy BE, Lembo AJ, Saito YA, Schiller LR, Soffer EE, Spiegel BM, Moayyedi P (2014) Efficacy of prebiotics, probiotics, and synbiotics in irritable bowel syndrome and chronic idiopathic constipation: systematic review and metaanalysis. Am J Gastroenterol 109(10):1547-1561. https://doi. org/10.1038/ajg.2014.202 (quiz 1546, 1562 prebiotes)

24. Tripathi A, Lammers KM, Goldblum S, Shea-Donohue T, Netzel-Arnett S, Buzza MS, Antalis TM, Vogel SN, Zhao A, Yang S, Arrietta MC, Meddings JB, Fasano A (2009) Identification of human zonulin, a physiological modulator of tight junctions, as prehaptoglobin-2. Proc Natl Acad Sci USA 106(39):1679916804. https://doi.org/10.1073/pnas.0906773106

25. Layer P, Andresen V, Pehl C, Allescher H, Bischoff SC, Classen M, Enck P, Frieling T, Haag S, Holtmann G, Karaus M, Kathemann S, Keller J, Kuhlbusch-Zicklam R, Kruis W, Langhorst J, Matthes H, Monnikes H, Muller-Lissner S, Musial F, Otto B, Rosenberger C, Schemann M, van der Voort I, Dathe K, Preiss JC (2011) Deutschen Gesellschaft fur Verdauungs- und S, Deutschen Gesellschaft fur Neurogastroenterologie und M [Irritable bowel syndrome: German consensus guidelines on definition, pathophysiology and management]. Z Gastroenterol 49(2):237-293. https://doi.org/10.1055/s-0029-1245976

26. Francis CY, Morris J, Whorwell PJ (1997) The irritable bowel severity scoring system: a simple method of monitoring irritable bowel syndrome and its progress. Aliment Pharmacol Ther 11(2):395-402

27. Badami E, Sorini C, Coccia M, Usuelli V, Molteni L, Bolla AM, Scavini M, Mariani A, King C, Bosi E, Falcone M (2011) Defective differentiation of regulatory FoxP3+ T cells by smallintestinal dendritic cells in patients with type 1 diabetes. Diabetes 60(8):2120-2124. https://doi.org/10.2337/db10-1201

28. Mann ER, Landy JD, Bernardo D, Peake ST, Hart AL, Al-Hassi HO, Knight SC (2013) Intestinal dendritic cells: their role in intestinal inflammation, manipulation by the gut microbiota and differences between mice and men. Immunol Lett 150(1-2):30-40. https://doi.org/10.1016/j.imlet.2013.01.007

29. Moser AM, Spindelboeck W, Strohmaier H, Enzinger C, Gattringer T, Fuchs S, Fazekas F, Gorkiewicz G, Wurm P, Hogenauer C, Khalil M (2017) Mucosal biopsy shows immunologic changes of the colon in patients with early MS. Neurol Neuroimmunol Neuroinflamm 4(4):e362. https://doi.org/10.1212/NXI.00000 00000000362

30. Klymiuk I, Bambach I, Patra V, Trajanoski S, Wolf P (2016) 16S based microbiome analysis from healthy subjects' skin swabs stored for different storage periods reveal phylum to genus level changes. Front Microbiol 7:2012. https://doi.org/10.3389/fmicb .2016.02012

31. Baker GC, Smith JJ, Cowan DA (2003) Review and re-analysis of domain-specific 16S primers. J Microbiol Methods 55(3):541-555

32. Schloss PD, Westcott SL, Ryabin T, Hall JR, Hartmann M, Hollister EB, Lesniewski RA, Oakley BB, Parks DH, Robinson CJ, 
Sahl JW, Stres B, Thallinger GG, Van Horn DJ, Weber CF (2009) Introducing mothur: open-source, platform-independent, community-supported software for describing and comparing microbial communities. Appl Environ Microbiol 75(23):7537-7541. https ://doi.org/10.1128/AEM.01541-09

33. Kozich JJ, Westcott SL, Baxter NT, Highlander SK, Schloss PD (2013) Development of a dual-index sequencing strategy and curation pipeline for analyzing amplicon sequence data on the MiSeq Illumina sequencing platform. Appl Environ Microbiol 79(17):5112-5120. https://doi.org/10.1128/AEM.01043-13

34. Wang Q, Garrity GM, Tiedje JM, Cole JR (2007) Naive Bayesian classifier for rapid assignment of rRNA sequences into the new bacterial taxonomy. Appl Environ Microbiol 73(16):5261-5267. https://doi.org/10.1128/AEM.00062-07

35. Caporaso JG, Bittinger K, Bushman FD, DeSantis TZ, Andersen GL, Knight R (2010) PyNAST: a flexible tool for aligning sequences to a template alignment. Bioinformatics 26(2):266-267. https://doi.org/10.1093/bioinformatics/btp636

36. Lozupone C, Lladser ME, Knights D, Stombaugh J, Knight R (2011) UniFrac: an effective distance metric for microbial community comparison. ISME J 5(2):169-172. https://doi.org/10.1038/ ismej.2010.133

37. Chao A (1984) Nonparametric estimation of the number of classes in a population. Scand J Stat 11:265-270

38. Segata N, Izard J, Waldron L, Gevers D, Miropolsky L, Garrett WS, Huttenhower C (2011) Metagenomic biomarker discovery and explanation. Genome Biol 12(6):R60. https://doi.org/10.1186/ gb-2011-12-6-r60

39. Immundiagnostik AGS-Aa, Bensheim 64625, Germany (2017) For the in vitro determination of zonulin in stool. http://www. immundiagnostik.com/fileadmin/pdf/Zonulin_K5600.pdf. Accessed 30 July 2018

40. Fasano A (2012) Intestinal permeability and its regulation by zonulin: diagnostic and therapeutic implications. Clin Gastroenterol Hepatol 10(10):1096-1100. https://doi.org/10.1016/j. cgh.2012.08.012

41. Shokryazdan P, Faseleh Jahromi M, Navidshad B, Liang JB (2016) Effects of prebiotics on immune system and cytokine expression. Med Microbiol Immunol. https://doi.org/10.1007/ s00430-016-0481-y

42. Sakaguchi S, Vignali DA, Rudensky AY, Niec RE, Waldmann H (2013) The plasticity and stability of regulatory T cells. Nat Rev Immunol 13(6):461-467. https://doi.org/10.1038/nri3464
43. Chadwick VS, Chen W, Shu D, Paulus B, Bethwaite P, Tie A, Wilson I (2002) Activation of the mucosal immune system in irritable bowel syndrome. Gastroenterology 122(7):1778-1783

44. Ligocki AJ, Niederkorn JY (2015) Advances on non-CD4+ Foxp $3+\mathrm{T}$ regulatory cells: CD8+, type 1 , and double negative $\mathrm{T}$ regulatory cells in organ transplantation. Transplantation 99(8):1553-1559. https://doi.org/10.1097/TP.000000000000081 3

45. Berni Canani R, Sangwan N, Stefka AT, Nocerino R, Paparo L, Aitoro R, Calignano A, Khan AA, Gilbert JA, Nagler CR (2015) Lactobacillus rhamnosus GG-supplemented formula expands butyrate-producing bacterial strains in food allergic infants. ISME J. https://doi.org/10.1038/ismej.2015.151

46. Ferrario C, Taverniti V, Milani C, Fiore W, Laureati M, De Noni I, Stuknyte M, Chouaia B, Riso P, Guglielmetti S (2014) Modulation of fecal Clostridiales bacteria and butyrate by probiotic intervention with Lactobacillus paracasei DG varies among healthy adults. J Nutr 144(11):1787-1796. https://doi.org/10.3945/ jn.114.197723

47. Furusawa Y, Obata Y, Fukuda S, Endo TA, Nakato G, Takahashi D, Nakanishi Y, Uetake C, Kato K, Kato T, Takahashi M, Fukuda NN, Murakami S, Miyauchi E, Hino S, Atarashi K, Onawa S, Fujimura Y, Lockett T, Clarke JM, Topping DL, Tomita M, Hori S, Ohara O, Morita T, Koseki H, Kikuchi J, Honda K, Hase K, Ohno H (2013) Commensal microbe-derived butyrate induces the differentiation of colonic regulatory $\mathrm{T}$ cells. Nature 504(7480):446-450. https://doi.org/10.1038/nature12721

48. Kelly CJ, Zheng L, Campbell EL, Saeedi B, Scholz CC, Bayless AJ, Wilson KE, Glover LE, Kominsky DJ, Magnuson A, Weir TL, Ehrentraut SF, Pickel C, Kuhn KA, Lanis JM, Nguyen V, Taylor CT, Colgan SP (2015) Crosstalk between microbiota-derived short-chain fatty acids and intestinal epithelial HIF augments tissue barrier function. Cell Host Microbe 17(5):662-671. https:// doi.org/10.1016/j.chom.2015.03.005

49. Elamin EE, Masclee AA, Dekker J, Pieters HJ, Jonkers DM (2013) Short-chain fatty acids activate AMP-activated protein kinase and ameliorate ethanol-induced intestinal barrier dysfunction in Caco-2 cell monolayers. J Nutr 143(12):1872-1881. https://doi. org/10.3945/jn.113.179549 